\title{
Effects of Furosemide on Cochlear Neural Activity, Central Hyperactivity and Behavioural Tinnitus after Cochlear Trauma in Guinea Pig
}

\author{
Wilhelmina H. A. M. Mulders*, Kristin M. Barry, Donald Robertson
}

The Auditory Laboratory, School of Anatomy, Physiology and Human Biology, The University of Western Australia, Crawley, Western Australia, Australia

\begin{abstract}
Cochlear trauma causes increased spontaneous activity (hyperactivity) to develop in central auditory structures, and this has been suggested as a neural substrate for tinnitus. Using a guinea pig model we have previously demonstrated that for some time after cochlear trauma, central hyperactivity is dependent on peripheral afferent drive and only later becomes generated intrinsically within central structures. Furosemide, a loop diuretic, reduces spontaneous firing of auditory afferents. We investigated in our guinea pig model the efficacy of furosemide in reducing 1) spontaneous firing of auditory afferents, using the spectrum of neural noise (SNN) from round window recording, 2) hyperactivity in inferior colliculus, using extracellular single neuron recordings and 3) tinnitus at early time-points after cochlear trauma. Tinnitus was assessed using gap prepulse inhibition of acoustic startle (GPIAS). Intraperitoneal furosemide, but not saline, caused a marked decrease in both SNN and central hyperactivity. Intracochlear perfusion with furosemide similarly reversed central hyperactivity. In animals in which GPIAS measurements suggested the presence of tinnitus (reduced GPIAS), this could be reversed with an intraperitoneal injection with furosemide but not saline. The results are consistent with furosemide reducing central hyperactivity and behavioural signs of tinnitus by acting peripherally to decrease spontaneous firing of auditory afferents. The data support the notion that hyperactivity may be involved in the generation of tinnitus and further suggest that there may be a therapeutic window after cochlear trauma using drug treatments that target peripheral spontaneous activity.
\end{abstract}

Citation: Mulders WHAM, Barry KM, Robertson D (2014) Effects of Furosemide on Cochlear Neural Activity, Central Hyperactivity and Behavioural Tinnitus after Cochlear Trauma in Guinea Pig. PLoS ONE 9(5): e97948. doi:10.1371/journal.pone.0097948

Editor: Berthold Langguth, University of Regensburg, Germany

Received January 22, 2014; Accepted April 27, 2014; Published May 16, 2014

Copyright: (c) 2014 Mulders et al. This is an open-access article distributed under the terms of the Creative Commons Attribution License, which permits unrestricted use, distribution, and reproduction in any medium, provided the original author and source are credited.

Funding: Supported by grants from the Royal National Institute for Deaf People (UK) G55, the Medical Health and Research Infrastructure Fund (WA) and The University of Western Australia. The funders had no role in study design, data collection and analysis, decision to publish, or preparation of the manuscript.

Competing Interests: The authors have declared that no competing interests exist.

*E-mail: helmy.mulders@uwa.edu.au

\section{Introduction}

A common side-effect of hearing loss is tinnitus, a phantom hearing sensation described as hissing or ringing in the ears [1]. Estimates of the prevalence of chronic tinnitus range from 10 to $15 \%$ of the adult population [2-5] but the incidence rises sharply in specific groups such as the elderly, workers in noisy environments and war veterans [6,7]. In about $20 \%$ of sufferers, tinnitus significantly affects daily life [8]. A number of previous studies in humans have suggested that the loop diuretic, furosemide may reduce tinnitus in some sufferers [9-11]. The present paper investigates the possible physiological mechanism of such an action.

Although many human studies have described abnormal activity within auditory pathways of tinnitus sufferers [12-15], the exact neural substrate is unknown. Animal models of hearing loss have shown increased spontaneous firing rates in central auditory structures (hyperactivity), alterations in neural synchrony, as well as reorganization [16-21], but exactly how these changes contribute to the development of tinnitus is still debated.

Because primary auditory afferents do not show increased spontaneous firing rates after common types of acoustic trauma $[1,18,22]$, it is often assumed that central hyperactivity is generated intrinsically and is not dependent on peripheral cochlear activity $[1,23,24]$. However, using a guinea pig model of acoustic trauma, we have shown that treatments that eliminate or reduce primary auditory nerve firing (cochlear ablation, cochlear cooling or cochlear perfusion with $\mathrm{CoCl}_{2}$ ), can significantly reduce hyperactivity in inferior colliculus [18,25]. Interestingly, this reduction of hyperactivity could only be fully achieved within the first 6 weeks after trauma, but not at later recovery times [26]. Based on these findings we have hypothesized that there are two distinct stages following cochlear trauma. In the first stage, central hyperactivity is the result of hyperexcitability of central neurons and is still dependent on peripheral afferent drive. This drive comprises the spontaneous firing of surviving primary afferent neurons, which is still present despite the fact that acoustic trauma reduces sensitivity to sound. In the second stage, central neurons become so excitable that they begin to generate their own intrinsic spontaneous firing and hyperactivity therefore becomes relatively independent of peripheral afferent input [27]. If hyperactivity is involved in the development of tinnitus, this suggests there may be a therapeutic window for recent-onset tinnitus in the first stage, using treatments that reduce cochlear afferent firing.

Furosemide is known to reduce primary auditory nerve firing [28]. Therefore in the present study we investigated, in our guinea 
pig model of cochlear trauma, the effect of furosemide on spontaneous firing of the auditory afferents, on hyperactivity in inferior colliculus and on behavioural measures of tinnitus.

\section{Materials and Methods}

\section{Ethics Statement}

The experimental protocols were approved by the Animal Ethics Committee of The University of Western Australia (03/ 100/1007) and were carried out in accordance with the Guidelines from the National Health and Medical Research Council Australia regarding the care and use of animals for experimental procedures. All surgery was performed under anaesthesia and all efforts were made to minimize suffering.

\section{Animals}

Thirty-two young adult pigmented guinea pigs of either sex were used. Animals (Tricolor strain) were derived from a local breeding colony at the University of Western Australia. Twenty of these animals were used to assess the therapeutic effects of intraperitoneal injection of furosemide $(80 \mathrm{mg} / \mathrm{kg})$ on tinnitus. The remaining twelve animals, weighing between 255 and $395 \mathrm{~g}$ at the time of acoustic trauma (10 kHz $124 \mathrm{~dB}$ SPL, 2 hours), were used to assess the effect of furosemide on spontaneous activity of the auditory nerve and on central hyperactivity measured in the central nucleus of the inferior colliculus (CNIC) 2 weeks after acoustic trauma. In eight of these animals furosemide $(n=4)$ or saline $(n=4)$ was administered intraperitoneally and in the remaining four animals furosemide was administered by intracochlear perfusion (see Fig. 1A).

\section{Behavioural Analysis for Tinnitus: GPIAS and PPI}

Behavioural testing for tinnitus consisted of gap prepulse inhibition of acoustic startle (GPIAS) and Prepulse Inhibition (PPI). GPIAS is a variation of PPI. PPI occurs when a weaker prestimulus, or prepulse, inhibits the reaction to a stronger stimulus. GPIAS consists of a comparison between two conditions. Both conditions consist of a background noise and startle pulse which elicits a startle response. However, in one condition, there is a gap within the continuous background noise which precedes the startle pulse. The gap in this case works as a prepulse. In normal animals, this condition results in inhibition of the startle response. It is thought that animals experiencing tinnitus that is qualitatively similar to the background noise, show decreased startle inhibition, i.e. deficits of GPIAS, because the tinnitus "fills in" the gap in the background noise $[29,30]$. Turner et al. compared GPIAS tests to other behavioural animal models of tinnitus and showed a strong positive correlation between the different methods [30].

A deficit in the GPIAS test could also be due to hearing loss (when an animal does not hear the background noise it cannot detect a gap therein) and therefore a PPI test is performed in parallel to the GPIAS test, using for the prepulse the same parameters as for the background noise in order to establish that the animal can hear the prepulse/background noise. Therefore, animals that fail GPIAS testing but pass PPI testing are thought to have tinnitus $[29,30]$.

Behavioural tests were performed on 20 animals. Seventeen animals, weighing between 332 and $649 \mathrm{~g}$ at the time of surgery, were exposed to a unilateral acoustic trauma in order to induce a hearing loss and tinnitus. The remaining 3 animals showed a GPIAS deficit before any cochlear trauma was performed and were therefore discarded from this study.

For behavioural testing, animals were mildly restrained in a clear polycarbonate holder which was placed on a custom- designed force transducer within a dark soundproof room. Just above the animal's head, two speakers were placed, one to administer the startle stimulus (Radio Shack 401278B; $115 \mathrm{~dB}$ SPL, narrowband noise, centre frequency $1 \mathrm{kHz}$, bandwidth $100 \mathrm{~Hz}, 20 \mathrm{~ms}$ duration, $0.1 \mathrm{~ms}$ rise/fall time,) and the other one for continuous background noise (for GPIAS) or a prepulse sound stimulus (for PPI) (Beyer DT 48). Custom-designed software (kindly provided by R. Salvi and D. Stolzberg) and commercial hardware (Tucker-Davis technologies) was used to deliver sound stimuli and record output from the custom-designed startle platform. Acoustic stimuli were calibrated using a $1 / 2$ " microphone positioned at the location of the animal's external ear canal.

Background noise for GPIAS consisted of a narrowband noise centred at either 8 or $14 \mathrm{kHz}(3 \mathrm{~dB}$ bandwidths $=1 \mathrm{kHz})$. These two frequency bands were chosen to fall within the centre of peripheral hearing loss $(14 \mathrm{kHz})$ and just below the region of hearing loss $(8 \mathrm{kHz})$. The $14 \mathrm{kHz}$ region is also the region of highest hyperactivity [31] in line with observations in human tinnitus subjects that the tinnitus pitch shows a strong correlation with the frequency region of hearing loss [32]. Intensity could be set at either 60 or $70 \mathrm{~dB}$ SPL. For each individual animal the lowest of these two intensity settings for which significant GPIAS ( $p<0.05$, see below: data analysis) would occur was determined and this level was then used throughout further testing for each animal. In the PPI tests the characteristics of the prepulse for PPI were identical to the background noise characteristics for GPIAS. Animals had to pass GPIAS and PPI twice before a cochlear trauma was performed.

A behavioural test consisted of 50 presentations of the startle stimulus with varying intervals $(20-30 \mathrm{~s})$ between presentations. Each test consisted of either the 8 or $14 \mathrm{kHz}$ background noise (GPIAS) or pre-pulse stimulus (PPI). During GPIAS testing the startle stimulus was embedded in the continuous background noise and half of the trials contained a $50 \mathrm{~ms}$ gap which preceded the startle stimulus by $100 \mathrm{~ms}$ (ISI $50 \mathrm{~ms}$ ). The order of gap (G) and no gap (NG) trials was randomized. During PPI testing the startle stimulus was embedded in silence, and a $50 \mathrm{~ms}$ prepulse was presented in half of the trials, but otherwise the PPI test was identical to the GPIAS test. A noise floor test was also performed to obtain a recording of the background level of movement for each animal (20 trials, with no startle or background noise present).

Each testing session contained three behavioural tests. First each animal was allowed to habituate in the soundproof room for $5 \mathrm{~min}$ before commencement of testing. Then the first test (50 trials see above) was either a GPIAS or PPI test, using either 8 or $14 \mathrm{kHz}$ background noise or prepulse, respectively. The second test was a noise floor test to ensure that all startle data were above the noise floor in all experiments. Then the third test was again either a GPIAS or PPI test, using the alternate frequency background noise or prepulse, respectively. The order of using 8 and $14 \mathrm{kHz}$ background noise/prepulse was alternated between sessions. There was at least one day between testing sessions. Only one testing session was conducted per day for each animal, with the only exception being the day of treatment. On this day the treatment testing session was conducted 1 hour after the previous session. No animal went through more than three testing sessions in one week in order to reduce the possibility of habituation and acclimatization $[29,33,34]$.

After each test it was determined whether an animal had failed or passed the GPIAS or PPI test. A Mann-Whitney statistical test was applied comparing startle amplitudes with and without GAP or pre-pulse. The animal was deemed to have failed the test when there was no significant difference between the gap/prepulse and 

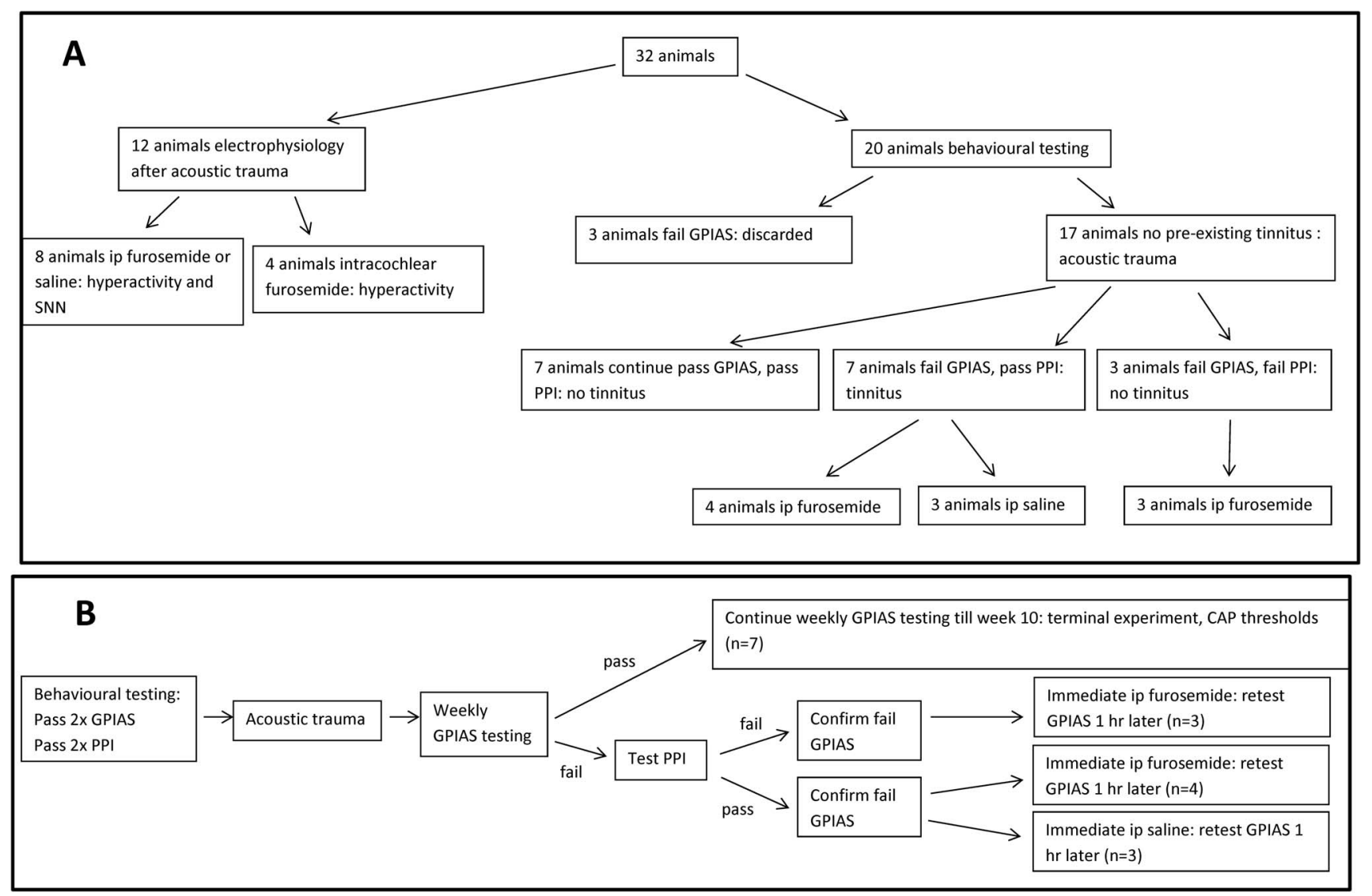

Figure 1. A: Overview of all animals used in the present study as allocated to the different groups. B: Schematic representation of the experimental design of behavioural experiments. doi:10.1371/journal.pone.0097948.g001

no gap/no prepulse conditions. Passing the test was characterized by a significant difference (significance level $\mathrm{p}<0.05$ ) between the two conditions.

After cochlear trauma, weekly GPIAS testing resumed. When an animal passed the GPIAS test the weekly testing continued. When an animal failed the GPIAS testing (i.e. no significant GPIAS), a PPI test was performed. If the animal passed the PPI test $(p<0.05)$ then two days later another GPIAS test was performed. If the animal failed this again and was categorized as a tinnitus animal, it immediately received an i.p. injection of either furosemide $(80 \mathrm{mg} / \mathrm{kg})$ or an equivalent amount of saline and was tested again (GPIAS) 1 hour later to assess the effect of treatment on behavioural signs of tinnitus. Three of the animals failed the PPI test as well as the GPIAS test (i.e. no significant pre pulse inhibition) and they also received an immediate i.p. injection of furosemide $(80 \mathrm{mg} / \mathrm{kg})$ and were tested again for GPIAS 1 hour later. After the treatment testing, animals underwent a final experiment, at least 3 days later, during which cochlear thresholds on both sides were measured.

\section{Recovery Surgery for Acoustic Trauma}

After a subcutaneous injection of $0.1 \mathrm{ml}$ atropine sulphate $(0.6 \mathrm{mg} / \mathrm{ml})$, animals received an intraperitoneal injection of Diazepam $(5 \mathrm{mg} / \mathrm{kg})$, followed 20 minutes later by an intramuscular injection of Hypnorm $(0.315 \mathrm{mg} / \mathrm{ml}$ fentanyl citrate and $10 \mathrm{mg} / \mathrm{ml}$ fluanisone; $1 \mathrm{ml} / \mathrm{kg}$ ). When deep anaesthesia was obtained as determined by the absence of the foot withdrawal reflex, the area of incision was shaved and animals were placed on a heating blanket in a soundproof room and mounted in hollow ear bars. A small opening (approximately $1 \mathrm{~mm}^{2}$ ) was made in the bulla and an insulated silver wire was placed on the round window. A compound action potential (CAP) audiogram [35] for the frequency range $4-24 \mathrm{kHz}$ was recorded to assess the animals' cochlear sensitivity. All sound stimuli were presented in a closed sound system through a $1 / 2$ " condenser microphone driven in reverse as a speaker (Bruel and Kjaer, type 4134). The system was calibrated using a $1 / 8^{\prime \prime}$ condensor microphone in place of the tympanic membrane and an absolute sound calibrator (Bruel and Kjaer type 4231). Pure tone stimuli (10 ms duration, $1 \mathrm{~ms}$ rise/fall times) were synthesized by a computer equipped with a DIGI 96 soundcard connected to an analog/digital interface (ADI-9 DS, RME Intelligent Audio Solution). Sample rate was $96 \mathrm{kHz}$. The interface was driven by a custom-made computer program (Neurosound, MI Lloyd), which was also used to collect single neuron data during the final experiments. CAP signals were amplified (1000x), filtered (100 Hz-3 kHz bandpass) and recorded with a second data acquisition system (Powerlab 4SP, AD Instruments).

When cochlear sensitivity was within the normal range [35], animals received a unilateral acoustic trauma using the closed sound system described above in the left cochlea. For this purpose animals were exposed to a continuous loud tone for $2 \mathrm{~h}(10 \mathrm{kHz}$, $124 \mathrm{~dB}$ SPL), whilst still anaesthetized. The contralateral ear was blocked with plasticine. Immediately after the acoustic trauma another CAP audiogram was measured, the incision was sutured and buprenorphin $(0.05 \mathrm{mg} / \mathrm{kg}$ subcutaneously) was given post- 
operatively as analgesic. Survival time varied between 2 (electrophysiological experiments) and 10 weeks (behavioural experiments).

\section{Surgery for Final Experiments}

Animals received a subcutaneous injection with $0.1 \mathrm{ml}$ atropine followed by an intraperitoneal injection of Nembutal (pentobarbitone sodium, $30 \mathrm{mg} / \mathrm{kg}$ ) and a $0.15 \mathrm{ml}$ intramuscular injection of Hypnorm. Anaesthesia was maintained with full Hypnorm doses every $h$ and half doses of Nembutal every $2 \mathrm{~h}$. When deep anaesthesia was obtained as determined by the absence of the foot withdrawal reflex, the areas of incision were shaved and animals were placed on a heating blanket in a sound proof room and artificially ventilated on carbogen $\left(\begin{array}{lllll}95 \% & \mathrm{O}_{2} & \text { and } & 5 \% & \mathrm{CO}_{2}\end{array}\right)$. Paralysis was induced with $0.1 \mathrm{ml}$ pancuronium bromide $(2 \mathrm{mg}$ / $\mathrm{ml}$ intramuscularly). The electrocardiogram was continuously monitored and heart rate never increased over pre-paralysis levels at any stage of the experiments. After the animals were mounted in hollow ear bars, the left and right cochleae were exposed and CAP audiograms were recorded on both sides with a silver wire placed on the round window as for the recovery procedures. Animals that had been used to assess effects of furosemide on GPIAS were then immediately euthanized with an injection of $0.3 \mathrm{ml}$ Lethabarb (sodium pentobarbitone $325 \mathrm{mg} / \mathrm{ml}$; VIRBAC).

During experiments testing the effects of a single acute intraperitoneal injection of furosemide or saline on central nerve activity, the spontaneous activity of the peripheral auditory nerve fibres was also assessed using the established technique of the spectrum of the neural noise (SNN) recorded from the round window of the noise-damaged cochlea. The latter recording has a prominent peak at approximately $900 \mathrm{~Hz}$ which can be used as a measure of the spontaneous activity of the primary afferent fibres $[36,37]$. To quantify the size of the $900 \mathrm{~Hz}$ peak of the SNN, the amplitude of the spectrum between 700 and $1100 \mathrm{~Hz}$ was averaged (10 averages/time point).

To obtain extracellular single neuron recordings in the central nucleus of the inferior colliculus (CNIC) a small craniotomy (approximately $4 \mathrm{~mm}^{2}$ ) overlying the visual cortex was performed and a glass-insulated tungsten microelectrode [38] was advanced using a stepping motor microdrive along the dorso-ventral axis through the cortex into the CNIC contralateral to the cochlea subjected previously to acoustic trauma. Electrode placement in the CNIC (about 2.5 to $3 \mathrm{~mm}$ ventral to the cortical surface) was indicated by the presence of strong sound-driven activity with a short latency (cluster onset latencies $<6.5 \mathrm{~ms}$ ) and a systematic progression from low to high characteristic frequencies (CF) with increasing depth. We have previously confirmed histologically that these response properties correlate with location of the electrode in the CNIC [31]. The craniotomy was covered with 5\% agar in saline to improve mechanical stability. When a single neuron was isolated its $\mathrm{CF}$ and threshold at $\mathrm{CF}$ were determined audio-visually and depth from the cortical surface was recorded using methods described previously [25,39]. In all neurons the spontaneous firing rate was measured for a period of $10 \mathrm{~s}$ as previously reported using an identical animal model [18,25,31].

\section{Effects of Furosemide on Central and Peripheral Nerve Activity}

To assess the effect of an acute i.p. injection with furosemide (Ilium, Australia, $50 \mathrm{mg} / \mathrm{ml}$ furosemide), in 8 animals, spontaneous firing rates of CNIC neurons were collected from the frequency regions between 4 and $24 \mathrm{kHz}$ before (approximately 2 hours of recording) and after an i.p. injection with furosemide ( $\mathrm{n}=4$ animals $)$ or saline $(\mathrm{n}=4$ animals). These single neuron recordings were interleaved with measurements of the SNN at regular intervals (every 5 to 15 minutes). After furosemide was injected (80 mg/kg bodyweight i.p.), SNN was monitored until a significant reduction could be observed (between 20 and 30 minutes after injection). We applied the same waiting period (30 minutes) after the saline injections. From that moment single neuron recordings resumed for another 2 hours interleaved with measurements of SNN at regular intervals (every 5 to 15 minutes).

To assess the effect of intracochlear injection of furosemide, a neuron with a high spontaneous firing rate was isolated within the region of the GNIC that showed hyperactivity. Activity from this neuron was then recorded at regular intervals before, during and after the perfusion (recordings of single neurons lasted for up to 130 minutes-see figure $1 \mathrm{D}$ and $\mathrm{E}$ ). The thresholds to $\mathrm{CF}$ tones of the same neurons were also recorded at regular intervals to monitor effects of furosemide on thresholds (see figure 1D and E). For the perfusions, a hole was made in the cochlear apex with the use of a hooked pick and a small hole was made in the scala tympani wall of the basal cochlear turn using a syringe needle. Using a micromanipulator the tip of a glass perfusion pipette was then carefully inserted through the hole in the scala tympani wall. The perfusion pipette contained artificial perilymph $(127 \mathrm{mM}$ $\mathrm{NaCl}, 5 \mathrm{mM} \mathrm{KCl}, 1 \mathrm{mM} \mathrm{MgCl} 2.6 \mathrm{H} 2 \mathrm{O}, 1 \mathrm{mM} \mathrm{NaH} 2 \mathrm{PO} 4 . \mathrm{H} 2 \mathrm{O}$, $12 \mathrm{mM}$ NaHCO3, $11 \mathrm{mM}$ glucose and $2 \mathrm{mM} \mathrm{CaCl} 2$ at $\mathrm{pH} 7.4$ ) with furosemide $(0.5 \mathrm{mM}$ or $1 \mathrm{mM})$. Perfusion rate was 2 to $3 \mu \mathrm{l}$ / $\min$.

\section{Data Analysis}

To identify statistically significant differences in spontaneous firing rates before and after intraperitoneal injection with furosemide or saline, a Kruskall-Wallis test was used as the data was not normally distributed, followed by Dunn's multiple comparison tests. The same test was used to assess the effect of furosemide and saline on SNN. We performed statistical analysis at three time points, $\mathrm{t}=0$ (time of injection) and at $\mathrm{t}=20$ (between 15-25 min after injection) and $t=120$ (between 110 and $125 \mathrm{~min}$ after injection). For statistical analysis of the CAP threshold data a one-way ANOVA and Bonferroni's multiple comparison tests were used.

For analysis of each GPIAS and PPI test in each animal, a Mann-Whitney statistical test was used to compare startle amplitudes with and without gap or pre-pulse (significance level $\mathrm{p}<0.05)$. For analysis of group data for GPIAS, the percentage suppression in the gap $(\mathrm{G})$ condition compared to the no gap $(\mathrm{NG})$ condition was calculated for each test $(\%$ GPIAS $=(1-(\mathrm{G} /$ $\mathrm{NG})$ )*100). Repeated measures (RM) one-way and two-way ANOVAs were performed comparing the suppression at three different time-points (before acoustic trauma, after acoustic trauma just before drug or control treatment, and after drug or control treatment).

\section{Results}

\section{Behavioural Testing Data}

Figure 1 shows an overview of all animals and their allocation to the different groups described in detail below (Fig. 1A) as well as a schematic representation of the overall experimental design for the behavioural experiments (Fig. 1B).

Of the 17 animals that showed significant initial GPIAS and PPI and then underwent acoustic trauma, 7 did not develop consistent (twice in one week) GPIAS deficits. Instead, these animals showed robust GPIAS throughout a period of 10 weeks post-acoustic trauma. Their \% GPIAS is shown in figure $2 \mathrm{~A}$ in the presence of both 8 and $14 \mathrm{kHz}$ background noise before the acoustic trauma 
(black bars) and at 10 weeks post-acoustic trauma (white bars). The fact that these animals continued to pass GPIAS testing suggests they did not develop tinnitus throughout this 10 week period and in addition supports the notion that GPIAS is a robust and consistent phenomenon. The mean audiograms for these animals are shown in figure 2B. The mean cochlear thresholds did not show statistically significant elevations at 10 weeks post-acoustic trauma. This is most likely due to the large variations in the extent of threshold recovery between animals as illustrated in figure $2 \mathrm{C}$, that shows the CAP threshold losses for each individual animal.

Of the 17 animals that showed significant initial GPIAS and PPI and then underwent acoustic trauma, 10 animals did develop GPIAS deficits at time-points between 3 and 6 weeks after acoustic trauma. Nine of these animals developed the GPIAS deficit with $14 \mathrm{kHz}$ background noise and one of them with $8 \mathrm{kHz}$ background noise. They were randomly allocated to a treatment after they were separated on the basis of their PPI data. Seven of these 10 animals showed significant PPI despite the GPIAS deficit, supporting the notion that the GPIAS deficit was due to tinnitus, whereas the other 3 animals also developed a PPI deficit suggesting the GPIAS deficit may not have been due to tinnitus. Four of the 7 animals that showed evidence of tinnitus received an i.p. injection with furosemide and 3 with saline. All 4 animals that received an i.p. injection with furosemide showed individually a return of significant GPIAS, in contrast to the 3 animals that received an i.p. injection with saline. These two groups of animals with intact PPI and different treatments are shown in Figure 3A. Note that only the GPIAS outcomes for the background noise condition (i.e. 8 or $14 \mathrm{kHz}$ centred noise) that revealed the deficit are shown in the group data and this will be done throughout the remainder of this paper. Statistical analysis revealed a significant interaction of time and treatment $(\mathrm{F}(2,10)=10.15, \mathrm{p}<0.01)$. Posthoc tests revealed that the animals which received furosemide showed significant $(\mathrm{p}<0.001)$ improvement in \% GPIAS when compared to those animals which received saline. All other comparisons between the two groups were not significant. These findings suggest that furosemide but not saline eliminates the behavioural signs of tinnitus.

Further statistical comparison indicated that within the furosemide treatment group, the \% GPIAS before acoustic trauma was significantly higher than after acoustic trauma $(p<0.001)$. Additionally, there was significantly less \% GPIAS after acoustic trauma than after treatment with furosemide $(\mathrm{p}<0.01)$. No other comparisons within the furosemide group were significant. Within the saline treated group, the \% GPIAS before acoustic trauma was significantly higher than both after acoustic trauma $(p<0.05)$ and after treatment with saline $(\mathrm{p}<0.01)$. No other comparisons within the saline group were significant. Mean CAP audiograms for these 7 animals that developed tinnitus are shown in figure 3C. Mean thresholds after recovery (varying between 4 and 7 weeks) were elevated compared to before acoustic trauma, but these changes were not statistically significant, most likely due to large interanimal variation (CAP threshold loss for each individual animal shown in Figure 3D). In addition, there were no statistically significant differences between the threshold loss observed in tinnitus animals compared to the threshold loss observed in nontinnitus animals.

The remaining 3 acoustic trauma animals that showed GPIAS deficits, also showed PPI deficits, which suggests that their GPIAS deficit may not have been due to tinnitus. These animals also received an i.p. injection with furosemide, but this had no effect on their GPIAS deficits as shown in figure 3B. In this figure the comparison is shown between the results from this group of animals with combined GPIAS deficit and PPI deficit and the
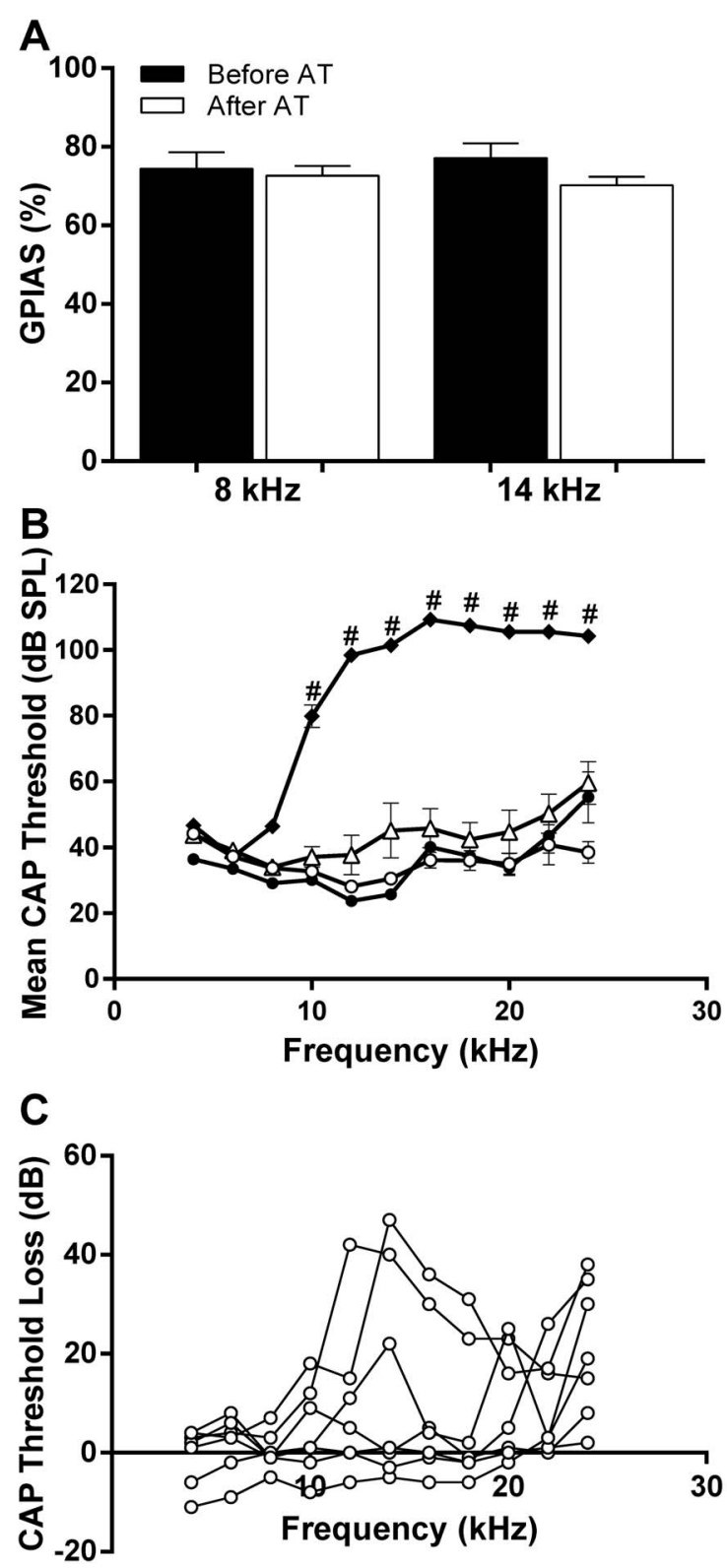

Figure 2. Data from 7 animals that underwent acoustic trauma and that did not develop a GPIAS deficit for a period of 10 weeks A: Histogram showing mean \% GPIAS. Shown is \% GPIAS measured with 8 and $14 \mathrm{kHz}$ background noise before (black bars) and 10 weeks after trauma (white bars). B: CAP thresholds (mean \pm SEM). Left cochlea before acoustic trauma (open circles), immediately after acoustic trauma (black diamonds) and after recovery from acoustic trauma (open triangles). Contralateral control cochlea after recovery (black circles). Statistical significance of differences between pre and post acoustic trauma data: $\# p<0.001$. C: Threshold loss for individual animals at 10 weeks after acoustic trauma.

doi:10.1371/journal.pone.0097948.g002

group of animals described above (GPIAS deficit but no PPI deficit). There was a significant interaction of time and PPI performance $(\mathrm{F}(2,10)=10.15, \mathrm{p}<.001)$. Post-hoc testing showed that after furosemide treatment, animals with impaired PPI showed significantly less \% GPIAS than those with intact PPI $(p<0.0001)$. These data show that when reduced GPIAS is associated with reduced PPI, furosemide cannot restore the suppression, unlike the situation when good PPI is still present. 
A

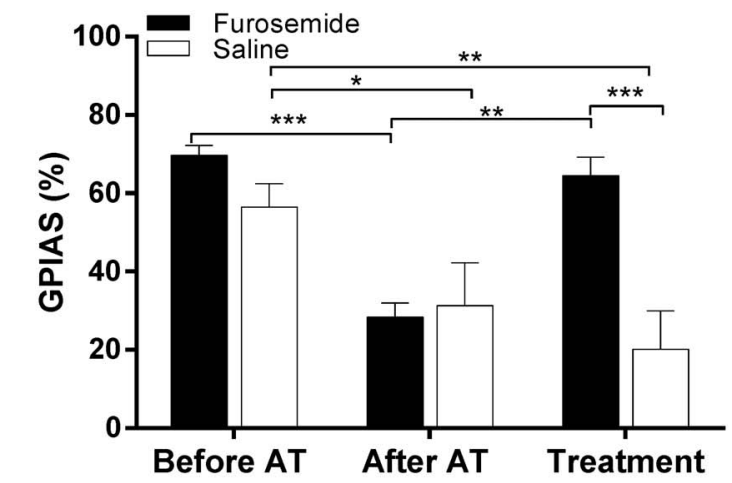

C

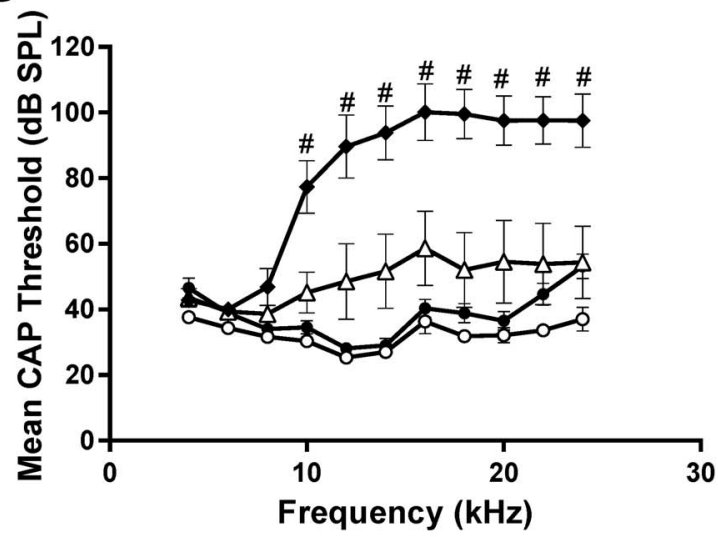

B
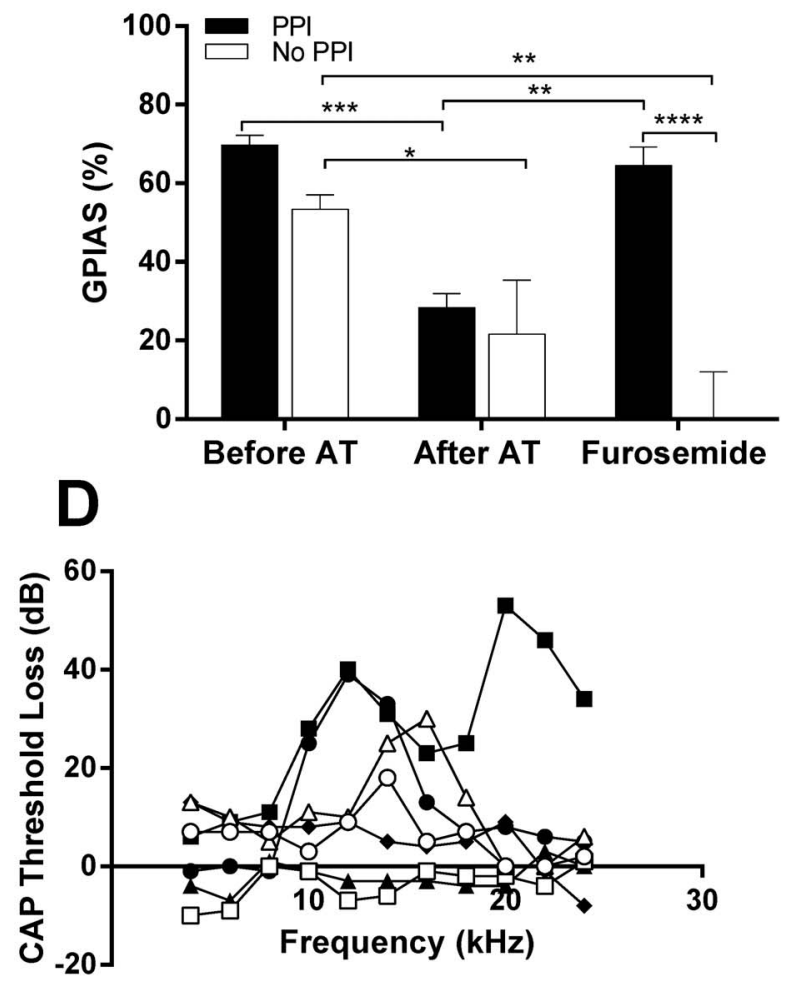

Figure 3. Data from animals that developed GPIAS deficits between 3 and 6 weeks post-trauma Histograms in A and B show \% GPIAS before acoustic trauma (AT), after AT and after treatment. A: Data from 4 animals given furosemide (black bars) and 3 animals given saline (white bars). All animals shown in A showed significant PPI. B: Data from 4 animals with significant PPI which were given furosemide (black bars, same animals as in panel A) and 3 animals without significant PPI which were also given furosemide (white bars). Significance is shown from repeated measure two-way ANOVA and post-tests. Statistical significance: ${ }^{*} p<0.05 ;{ }^{* *} p<0.01$. ${ }^{* *} p<0.001$. C: CAP thresholds from animals shown in A (mean \pm SEM). Left cochlea before acoustic trauma (open circles), immediately after acoustic trauma (black diamonds) and after recovery from acoustic trauma (open triangles). Contralateral control cochlea after recovery (black circles). Statistical significance of differences between pre and post acoustic trauma data: \#p<0.001. D: Threshold loss for individual animals shown in A. Black symbols are animals given furosemide and open symbols animals given saline.

doi:10.1371/journal.pone.0097948.g003

This shows that the beneficial action of furosemide is specific to those animals which may have tinnitus and it is therefore unlikely to be having a non-specific effect on startle circuitry per se.

Additional statistical comparisons between the intact PPI and impaired PPI animals (Fig. 3B), indicated that within the intact PPI group, the \% GPIAS before acoustic trauma was significantly higher than after acoustic trauma $(\mathrm{p}<0.01)$. Additionally, there was a significantly higher \% GPIAS after treatment with furosemide than after acoustic trauma $(p<0.01)$. No other comparisons within the intact PPI group were significant. Within the PPI deficit group, the \% GPIAS before acoustic trauma was greater than both after acoustic trauma $(\mathrm{p}<0.05)$ and after treatment with saline $(\mathrm{p}<0.01)$.

\section{Acute Effects of Furosemide on Peripheral and Central Neural Activity}

Figure $4 \mathrm{~A}$ shows the magnitude of the SNN measured from the round window in 8 different animals before and after i.p. injection with furosemide $(n=4)$ or saline $(n=4)$. The $\mathrm{SNN}$ can be used as a measure of spontaneous cochlear neural activity [36,37]. In all animals injected with furosemide, the SNN showed a large reduction, which reached a maximum (40 to $70 \%$ reduction) 20 to
30 minutes after injection. The SNN remained decreased up to $3 \mathrm{~h}$ after injection (the maximum period recorded) in 3 animals but showed recovery to $89 \%$ of its initial value in one animal after approximately 2 hours. In contrast, in animals injected with saline, the SNN remained stable. Statistical analysis showed no significant difference between the SNN in the furosemide group and the saline group at time of injection but a significant reduction of SNN after furosemide injection compared to saline injection at $\mathrm{t}=20 \mathrm{~min}$ and $\mathrm{t}=120 \mathrm{~min}(\mathrm{p}<0.05)$. These data show that i.p. administration of furosemide can decrease the spontaneous firing of the primary auditory nerve fibres.

The effects of i.p. injection of furosemide or saline on spontaneous firing rates of single neurons in CNIC, are illustrated in figure 4B. These data were obtained from 8 animals that were exposed to acoustic trauma 2 weeks previously and had therefore developed hyperactivity in CNIC (note that these animals were the same as used for the measurements of SNN described above). The mean spontaneous activity of sampled CNIC neurons before furosemide injection was $8.12 \pm 1.1$ spikes/sec (ranging from 0 to 74.4 spikes/sec; $n=172$ neurons from 4 animals), very similar to the mean spontaneous activity measured in 4 other animals before an injection with saline $(8.1 \pm 0.8$ spikes $/ \mathrm{sec}$, ranging from 0 to 

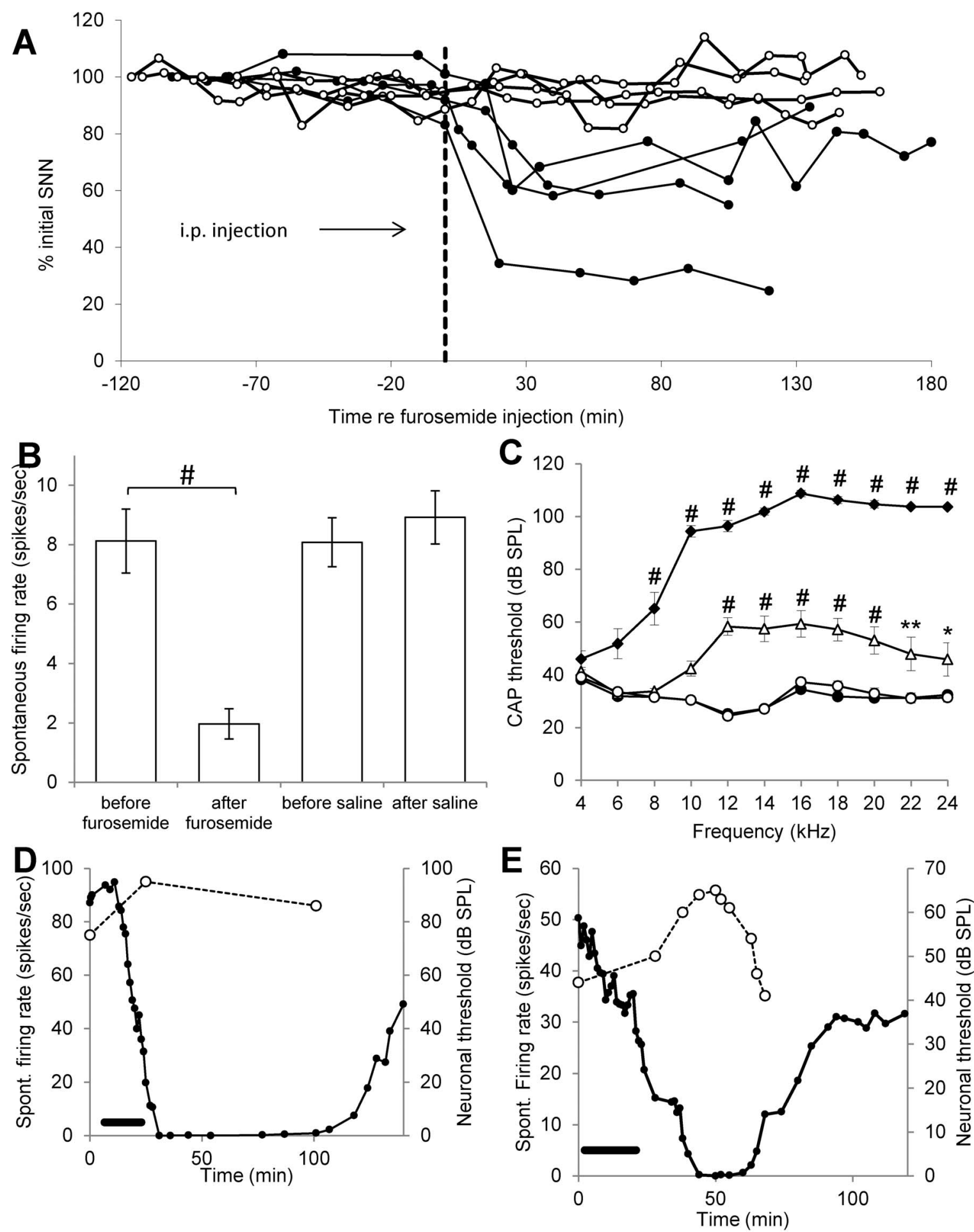

Figure 4. A: Spectrum of neural noise (SNN) recorded from the round window in 8 animals plotted as percentage of original value before and after an i.p. injection with furosemide (black line with filled circles $n=4$ ) or saline (black line with open circle $n=4$ ). Time of injection indicated by dotted line. B: Mean spontaneous firing rate of CNIC neurons recorded before and after i.p. furosemide and before and after saline $(n=4$ for each group; mean \pm SEM). C: CAP thresholds (mean \pm SEM) at different frequencies recorded from the left cochlea before acoustic trauma (open circles), immediately after acoustic trauma (black diamonds) and after recovery from acoustic trauma (open triangles), as well as from the contralateral control cochlea after recovery (black circles). ${ }^{*} p<0.05 ;{ }^{* *} p<0.01 ; \# p<0.001$ statistical significance as compared to before trauma data. D and E: Spontaneous firing rate of 2 individual neurons from 2 different animals plotted over time before, during and after intracochlear perfusion of furosemide (black circles). Black bar indicates timing of intracochlear perfusion. Open circles indicate measurement of the neuron's threshold to CF tones. Neuronal $\mathrm{CF}$ is $10 \mathrm{kHz}$ and $12.4 \mathrm{kHz}$, in $\mathrm{D}$ and $\mathrm{E}$, respectively.

doi:10.1371/journal.pone.0097948.g004 
75.9 spikes/sec; $\mathrm{n}=235$ neurons), indicating levels of neural hyperactivity in good agreement with those previously reported using identical methods [18,26]. After the injection with furosemide (and after a decrease was observed in the averaged SNN as shown in Fig. 4A) the mean spontaneous firing rates in CNIC decreased to $1.97 \pm 0.5$ spikes/sec (ranging from 0 to 44.8 spikes/ sec; $\mathrm{n}=179$ neurons from 4 animals). This decrease in the spontaneous firing rates after furosemide was statistically significant, both when comparing within each individual animal as well as when using the pooled group data (all $\mathrm{p}<0.001)$. In contrast, an injection with saline in the other 4 animals had no effect $(8.9 \pm 0.9$ spikes/sec (ranging from 0 to 77.2 spikes/sec; $n=225$ neurons). These data show that an acute i.p. injection with furosemide simultaneously reduced peripheral afferent spontaneous firing (SNN measurements) and lowered the hyperactive spontaneous firing rates seen in IC neurons two weeks after recovery from acoustic trauma.

We were able to record the effect of intracochlear perfusion of furosemide on 6 individual CNIC neurons that showed high spontaneous firing rates varying between 25 to 90 spikes/sec 2 weeks after acoustic trauma. In 5 of these neurons the spontaneous firing rate reduced to 0 spikes/sec and in the remaining neuron spontaneous firing rate reduced by $68.6 \%$ (from 34.7 to 10.9 spikes/sec). Four of the neurons were recorded from for long enough to observe partial recovery of spontaneous firing rate. The effect of intracochlear perfusion with furosemide on two of these neurons is illustrated in figures $4 \mathrm{D}$ and $4 \mathrm{E}$. In addition, in these figures the thresholds of these neurons to acoustic stimulation are shown (white open circles). These show that furosemide causes a relatively small increase in neuronal thresholds and that these seem to recover at a faster rate than the spontaneous firing rate (note that thresholds for the two neurons shown in Fig. 4D,E are rather high because these neurons emanate from cochlear regions that were subjected to acoustic trauma 2 weeks before recording). These data show that an intracochlear effect of furosemide is sufficient to cause suppression of the hyperactive spontaneous firing rates seen in IC neurons two weeks after recovery from acoustic trauma.

Figure 4C shows the cochlear CAP thresholds after recovery from acoustic trauma in the 8 animals used to describe the i.p. and intracochlear effects of furosemide on neural activity. The effects of acoustic trauma were as described previously $[18,31,40]$. Immediately after acoustic trauma all CAP thresholds $\geq 8 \mathrm{kHz}$ were significantly increased compared to before acoustic trauma values, but after 14 to 25 days (our recovery periods) thresholds recovered substantially and were only significantly different from pre-acoustic trauma values at frequencies $\geq 12 \mathrm{kHz}$ (one-way ANOVA $\mathrm{F}(32,231)=89.17, \mathrm{p}<0.0001$, details from post-hoc analysis shown in figure). No difference was observed between the noise-damaged (before trauma values) and contralateral control cochlear thresholds (measured after recovery from trauma), demonstrating that contralateral control cochlear thresholds remained unaffected by the cochlear trauma on the other side.

\section{Discussion}

This paper provides for the first time, direct evidence in a single animal model that a drug treatment that reduces spontaneous firing rates in the auditory nerve, eliminates the hyperactivity in the CNIC caused by acoustic trauma, and also eliminates the behavioural signs of tinnitus.

In agreement with previous studies, acute systemic furosemide caused a reduction of spontaneous firing rates of auditory nerve fibres [28], as demonstrated by decreased SNN [36,37]. This is most likely due to a decreased endocochlear potential, caused by an effect of furosemide on ion transporters in the stria vascularis and subsequent reduction of spontaneous neurotransmitter release from inner hair cells [28,41]. Also in agreement with previous descriptions of the peripheral action of furosemide, the effects on spontaneous cochlear afferent activity were accompanied by relatively minor changes in cochlear neural thresholds that recovered faster than the cochlear spontaneous firing rates. Sewell [28] showed in cats, that primary afferent spontaneous firing is more sensitive than thresholds to falls in endocochlear potential induced by furosemide. This suggests that furosemide, or related drugs, could be used to selectively reduce primary afferent spontaneous firing without major effects on hearing sensitivity, which could be beneficial for any future therapeutic application.

A major finding was that acute administration of furosemide also caused a marked reduction in the spontaneous hyperactivity that developed in the GNIC after acoustic trauma. These findings are in agreement with our previous studies showing an elimination of central hyperactivity after other treatments directly reducing cochlear neural output [18,25].

Finally, in animals with unequivocal behavioural evidence of tinnitus (reduced GPIAS and intact PPI), an i.p. injection with furosemide, but not saline, dramatically altered the result of GPIAS testing, showing a return of significant GPIAS, suggesting that furosemide reduced the level of tinnitus in these animals. The effect was robust in that all animals in this group showed a return of GPIAS after furosemide, whereas none did so after saline. Similar observations of the effectiveness of furosemide were made in 3 other animals in our laboratory that developed failures in GPIAS after mechanical lesion of the cochlea or that had preexisting GPIAS deficits before a trauma to the cochlea (data not shown). We have previously shown that mechanical lesions also results in hyperactivity in IC neurons [42]. A specific therapeutic effect of furosemide on tinnitus is further supported by the fact that 3 animals that showed a GPIAS deficit but also a PPI deficit, did not respond positively to furosemide administration, since in these animals the GPIAS deficit was most likely due to factors other than tinnitus (see below). This suggests that furosemide does not exert its effects via a general effect on acoustic startle.

Approximately $60 \%$ of animals subjected to acoustic trauma (10 out of 17 animals) developed repeatable deficits of GPIAS. A deficit of GPIAS can be an indication of tinnitus as GPIAS testing strongly correlates with other behavioural testing paradigms for tinnitus [30]. However, a deficit in GPIAS could also be a result of hearing loss, habituation or unknown startle circuitry deficits $[29,30,43]$ and we included the PPI test in our experiments in order to screen for these possibilities. Indeed, when also tested for PPI, $30 \%$ of these animals with GPIAS deficits (17\% of total), also showed a PPI deficit. The remaining animals with GPIAS deficits showed significant PPI and these latter animals $(41 \%$ of total numbers receiving acoustic trauma) could therefore be categorized as fulfilling criteria consistent with their experiencing tinnitus. Because the pre-pulse stimulus had the same acoustic characteristics as the background noise used in the GPIAS test, this provided valuable confirmation that the animals could still detect the background noise and that their startle circuitry was functioning normally. The percentage of animals showing tinnitus shows large variations between studies, i.e. in guinea pigs $(57 \% ; 4$ out of 7 animals) [29], in mice (50\%) [44] or in rats (between 30 and $75 \%$ ) [45-48]. This could be due to species differences or to the different parameters of cochlear trauma between different studies. The fact that not all animals develop signs of tinnitus is in 
agreement with human population data showing that not all individuals with a hearing loss develop tinnitus [2,4].

Taken together, our results seem to provide a mechanism for the possible therapeutic effect of furosemide in treating tinnitus, which has in fact been reported in human patients $[9,11,49]$. However, there are several important qualifications that must be placed on this conclusion.

First, the dose of furosemide $(80 \mathrm{mg} / \mathrm{kg})$ that we used for i.p injection is more than 10 times the usual hourly intravenous dose recommended in humans for treatment of severe renal and cardiovascular conditions, and it is higher than the oral dose used in the past for treatment of human tinnitus. However, direct comparison between human and guinea pig is not possible (for example different metabolic rates [50] might mean a lower dose could be used in human to produce therapeutic effects on tinnitus) and clearly more work is needed to elucidate whether lower doses that are suitable for human application can produce the physiological and behavioural effects we report here.

Second, the present study used only a single dose of furosemide and involved a single session of behavioural testing immediately after. Hence it is not known if the behavioural effects seen are long lasting or whether chronic administration of furosemide would be required to permanently suppress central hyperactivity and tinnitus.

Third, furosemide might affect CNIC hyperactivity via direct central actions, since the $\mathrm{Na}-\mathrm{K}-2 \mathrm{Cl}$ co-transporter found in the inner ear is also expressed centrally [51] and a central effect cannot be ruled out by the present experiments. However, the fact that we saw changes in peripheral neural activity after i.p furosemide and also observed complete elimination of spontaneous hyperactivity in GNIC neurons after local intracochlear injection, is compatible with the notion that an intracochlear action of furosemide is sufficient to induce the central effects.

Fourth, we have only investigated the effects of furosemide between 3-8 weeks after cochlear trauma. Our previous work showed that the peripheral dependence of central hyperactivity is temporary [18], and that the central-intrinsic phase emerges at

\section{References}

1. Eggermont JJ, Roberts LE (2004) The neuroscience of tinnitus. TRENDS in neuroscience 27: 676-682.

2. Shargorodsky J, Curhan GC, Farwell WR (2010) Prevalence and characteristics of tinnitus among US adults. Am J Med 123: 711-718.

3. Vio MM, Holme RH (2005) Hearing loss and tinnitus: 250 million people and a US\$10 billion potential market. Drug Discov Today 10: 1263-1265.

4. Axelsson A, Ringdahl A (1989) Tinnitus-a study of its prevalence and characteristics. Br J Audiol 23: 53-62.

5. Salvi R, Lobarinas E, Sun W (2009) Pharmacological treatments for tinnitus: new and old. Drugs Fut 34: 381-400.

6. Yankaskas K (2013) Prelude: noise-induced tinnitus and hearing loss in the military. Hear Res 295: 3-8.

7. Mrena R, Savolainen S, Kiukaanniemi H, Ylikoski J, Makitie AA (2009) The effect of tightened hearing protection regulations on military noise-induced tinnitus. Int J Audiol 48: 394 400.

8. Andersson G, Kaldo V (2004) Internet-based cognitive behavioral therapy for tinnitus. J Clin Psychol 60: 171-178.

9. Cesarani A, Capobianco S, Soi D, Giuliano DA, Alpini D (2002) Intratympanic dexamethasone treatment for control of subjective idiopathic tinnitus: our clinical experience. Int Tinnitus J 8: 111-114.

10. Nakai Y, Yamane H, Minowa Y, Go K, Fukumaru M, et al. (1982) Application of loop diuretics for treatment of sensorineural hearing impairment. Experimental and clinical study. Acta Otolaryngol 94: 37-43.

11. Risey JA, Guth PS, Amedee RG (1995) Furosemide Distinguishes Central and Peripheral Tinnitus. Int Tinnitus J 1: 99-103.

12. Lockwood AH, Salvi RJ, Burkard RF, Galantowicz PJ, Coad ML, et al. (1999) Neuroanatomy of tinnitus. Scand Audiol Suppl 51: 47-52.

13. Melcher JR, Sigalovsky IS, Guinan JJ, Jr., Levine RA (2000) Lateralized tinnitus studied with functional magnetic resonance imaging: abnormal inferior colliculus activation. J Neurophysiol 83: 1058-1072.

14. Lanting GP, de Kleine E, van Dijk P (2009) Neural activity underlying tinnitus generation: results from PET and fMRI. Hear Res 255: 1-13. around 8 to 12 weeks post-trauma [27]. At this latter stage, both hyperactivity and tinnitus should become resistant to furosemide treatment and further animal studies are needed to test this hypothesis. Nonetheless, our present data could provide a physiological explanation for the partial success of furosemide treatment in human tinnitus patients $[9,11,49]$. Risey et al. [11] reported that $50 \%$ of patients experienced alleviation of tinnitus after furosemide administration and we suggest that patients who did not respond to furosemide, had tinnitus that was in the second, central-intrinsic, phase. Cesarani et al. [9] showed acute positive effects of furosemide in $74 \%$ of patients, with increased effectiveness when treatment was given during the first 3 months after tinnitus onset. Others also showed greater beneficial effects of furosemide treatment in patients with fresh cases of deafness than old cases of deafness, again supporting our hypothesis [10].

Finally, although the major finding of this paper strengthens the notion of a link between central neural hyperactivity and tinnitus, this relationship is by no means clear. Hyperactivity in CNIC develops rapidly (within hours after cochlear trauma) [52], as does auditory cortex hyperactivity [19,53], but tinnitus in our animals was only observed a minimum of 3 weeks after cochlear trauma. This suggests that tinnitus development requires the involvement of other brain regions. Other brain regions that have been suggested to be involved include the limbic system and recently, the paraflocculus of the cerebellum $[54,55]$.

\section{Acknowledgments}

The authors thank R. Salvi and D. Stolzberg for generous sharing of software and advice.

\section{Author Contributions}

Conceived and designed the experiments: WHAMM KMB DR. Performed the experiments: WHAMM KMB DR. Analyzed the data: WHAMM KMB DR. Contributed reagents/materials/analysis tools: WHAMM DR. Wrote the paper: WHAMM KMB DR.

15. Gu JW, Halpin CF, Nam EC, Levine RA, Melcher JR (2010) Tinnitus, diminished sound-level tolerance, and elevated auditory activity in humans with clinically normal hearing sensitivity. J Neurophysiol 104: 3361-3370.

16. Eggermont JJ, Komiya $\mathrm{H}$ (2000) Moderate noise trauma in juvenile cats results in profound cortical topographic map changes in adulthood. Hear Res 142: 89101.

17. Finlayson PG, Kaltenbach JA (2009) Alterations in the spontaneous discharge patterns of single units in the dorsal cochlear nucleus following intense sound exposure. Hear Res 256: 104-117.

18. Mulders WH, Robertson D (2009) Hyperactivity in the auditory midbrain after acoustic trauma: dependence on cochlear activity. Neuroscience 164: 733-746.

19. Norena AJ, Eggermont JJ (2003) Changes in spontaneous neural activity immediately after an acoustic trauma: implications for neural correlates of tinnitus. Hear Res 183: 137-153.

20. Vogler DP, Robertson D, Mulders WH (2011) Hyperactivity in the ventral cochlear nucleus after cochlear trauma. J Neurosci 31: 6639-6645.

21. Robertson D, Irvine DR (1989) Plasticity of frequency organization in auditory cortex of guinea pigs with partial unilateral deafness. J Comp Neurol 282: 456471 .

22. Liberman MC, Dodds LW (1984) Single-neuron labeling and chronic cochlear pathology. II. Stereocilia damage and alterations of spontaneous discharge rates. Hear Res 16: 43-53.

23. Brozoski TJ, Bauer CA (2005) The effect of dorsal cochlear nucleus ablation on tinnitus in rats. Hear Res 206: 227-236.

24. Zacharek MA, Kaltenbach JA, Mathog TA, Zhang J (2002) Effects of cochlear ablation on noise induced hyperactivity in the hamster dorsal cochlear nucleus: implications for the origin of noise induced tinnitus. Hear Res 172: 137-143.

25. Mulders WH, Seluakumaran K, Robertson D (2010) Efferent pathways modulate hyperactivity in inferior colliculus. J Neurosci 30: 9578-9587.

26. Mulders WH, Robertson D (2011) Progressive centralization of midbrain hyperactivity after acoustic trauma. Neuroscience 192: 753-760. 
27. Robertson D, Bester C, Vogler D, Mulders WH (2013) Spontaneous hyperactivity in the auditory midbrain: relationship to afferent input. Hear Res 295: 124-129.

28. Sewell WF (1984) The relation between the endocochlear potential and spontaneous activity in auditory nerve fibres of the cat. J Physiol 347: 685-696.

29. Dehmel S, Eisinger D, Shore SE (2012) Gap prepulse inhibition and auditory brainstem-evoked potentials as objective measures for tinnitus in guinea pigs. Front Syst Neurosci 6: 42.

30. Turner JG, Brozoski TJ, Bauer CA, Parrish JL, Myers K, et al. (2006) Gap detection deficits in rats with tinnitus: a potential novel screening tool. Behav Neurosci 120: 188-195.

31. Mulders WH, Ding D, Salvi R, Robertson D (2011) Relationship between auditory thresholds, central spontaneous activity, and hair cell loss after acoustic trauma. J Comp Neurol 519: 2637-2647.

32. Norena A, Micheyl C, Chery-Croze S, Collet L (2002) Psychoacoustic characterization of the tinnitus spectrum: implications for the underlying mechanisms of tinnitus. Audiol Neurootol 7: 358-369.

33. Davis M (1974) Sensitization of the rat startle response by noise. J Comp Physiol Psychol 87: 571-581.

34. Faraday MM, Grunberg NE (2000) The importance of acclimation in acoustic startle amplitude and pre-pulse inhibition testing of male and female rats. Pharmacol Biochem Behav 66: 375-381.

35. Johnstone JR, Alder VA, Johnstone BM, Robertson D, Yates GK (1979) Cochlear action potential threshold and single unit thresholds. J Acoust Soc Am 65: 254-257.

36. McMahon CM, Patuzzi RB (2002) The origin of the $900 \mathrm{~Hz}$ spectral peak in spontaneous and sound-evoked round-window electrical activity. Hear Res 173: 134-152.

37. Patuzzi RB, Brown DJ, McMahon CM, Halliday AF (2004) Determinants of the spectrum of the neural electrical activity at the round window: transmitter release and neural depolarisation. Hear Res 190: 87-108.

38. Merrill EG, Ainsworth A (1972) Glass-coated platinum-plated tungsten microelectrodes. Med Biol Eng 10: 662-672.

39. Ingham NJ, Bleeck S, Winter IM (2006) Contralateral inhibitory and excitatory frequency response maps in the mammalian cochlear nucleus. Eur J Neurosci 24: 2515-2529.

40. Dong S, Mulders WH, Rodger J, Woo S, Robertson D (2010) Acoustic trauma evokes hyperactivity and changes in gene expression in guinea-pig auditory brainstem. Eur J Neurosci 31: 1616-1628.
41. Rybak LP, Morizono T (1982) Effect of furosemide upon endolymph potassium concentration. Hear Res 7: 223-231.

42. Dong S, Mulders WH, Rodger J, Robertson D (2009) Changes in neuronal activity and gene expression in guinea-pig auditory brainstem after unilateral partial hearing loss. Neuroscience 159: 1164-1174.

43. Lobarinas E, Hayes SH, Allman BL (2013) The gap-startle paradigm for tinnitus screening in animal models: limitations and optimization. Hear Res 295: 150160.

44. Middleton JW, Kiritani T, Pedersen C, Turner JG, Shepherd GM, et al. (2011) Mice with behavioral evidence of tinnitus exhibit dorsal cochlear nucleus hyperactivity because of decreased GABAergic inhibition. Proc Natl Acad Sci U S A 108: 7601-7606.

45. Zhang J, Zhang Y, Zhang X (2011) Auditory cortex electrical stimulation suppresses tinnitus in rats. J Assoc Res Otolaryngol 12: 185-201.

46. Wang H, Brozoski TJ, Turner JG, Ling L, Parrish JL, et al. (2009) Plasticity at glycinergic synapses in dorsal cochlear nucleus of rats with behavioral evidence of tinnitus. Neuroscience 164: 747-759.

47. Kraus KS, Mitra S, Jimenez Z, Hinduja S, Ding D, et al. (2010) Noise trauma impairs neurogenesis in the rat hippocampus. Neuroscience 167: 1216-1226.

48. Ruttiger L, Singer W, Panford-Walsh R, Matsumoto M, Lee SC, et al. (2013) The reduced cochlear output and the failure to adapt the central auditory response causes tinnitus in noise exposed rats. PLoS One 8: e57247.

49. Alpini D, Gesarani A, Giuliano DA, Capobianco S (2004) Tinnitus: pharmacological topodiagnosis. Int Tinnitus J 10: 91-93.

50. Gillooly JF, Brown JH, West GB, Savage VM, Charnov EL (2001) Effects of size and temperature on metabolic rate. Science 293: 2248-2251.

51. Blaesse P, Airaksinen MS, Rivera G, Kaila K (2009) Cation-chloride cotransporters and neuronal function. Neuron 61: 820-838.

52. Mulders WH, Robertson D (2013) Development of hyperactivity after acoustic trauma in the guinea pig inferior colliculus. Hear Res 298: 104-108.

53. Seki S, Eggermont JJ (2003) Changes in spontaneous firing rate and neural synchrony in cat primary auditory cortex after localized tone-induced hearing loss. Hear Res 180: 28-38.

54. Bauer CA, Kurt W, Sybert LT, Brozoski TJ (2013) The cerebellum as a novel tinnitus generator. Hear Res 295: 130-139.

55. De Ridder D, Elgoyhen AB, Romo R, Langguth B (2011) Phantom percepts: tinnitus and pain as persisting aversive memory networks. Proc Natl Acad Sci U S A 108: 8075-8080. 